\title{
EDITORIALS
}

\section{Cannabis use in remote Indigenous communities in Australia: endemic yet neglected}

\author{
KS Kylie Lee, Katherine M Conigrave, George C Patton and Alan R Clough \\ The effects of cannabis use on health and social adjustment are profound
}

S ubstance misuse by Indigenous people has long been recognised as one of the devastating consequences of contact with Western culture. Misuse of tobacco, alcohol and petrol among Indigenous Australians has received much attention. Cannabis, by contrast, has not been viewed as a major problem. But since the 1990s, it has become apparent that heavy cannabis use is common in some remote Indigenous communities. ${ }^{1}$ The associated health and social burdens are now being recognised. ${ }^{1,2}$

Indigenous Australians, whether living in urban or rural settings, are more likely than other Australians to report cannabis use. $^{3}$ Recent reports suggest that cannabis use is also relatively high among Indigenous populations in New Zealand, Canada and North America. ${ }^{4}$ Limited data are available on patterns of cannabis use among Indigenous Australians. ${ }^{3}$ However, a recent 5 -year study of adolescents and young adults in three remote communities in Arnhem Land in the Northern Territory has found that not only is cannabis use common in remote Indigenous settings, but its effects on health and social adjustment are profound. . $^{4-6}$

These three communities are close to one another but very isolated, being over 550 kilometres from the nearest city. There is one local Indigenous language, and English is a secondary language. Tobacco use was found to be the norm in these communities, with over $90 \%$ of adolescents and young adults smoking. ${ }^{7}$ Because of restricted access to alcohol, problem drinking was uncommon. ${ }^{7}$ In contrast, cannabis use was endemic, with over $70 \%$ of males and $20 \%$ of females being current users. ${ }^{5}$ Cannabis was typically consumed mixed with tobacco and smoked using a locally fashioned "bucket bong" that gives the user a rapid and intense dose with little smoke lost. ${ }^{5}$ Regular heavy use $(\geqslant 6$ "cones" daily) was found in almost $90 \%$ of users. ${ }^{7}$ This is around twice the consumption of regular cannabis users elsewhere in Australia. ${ }^{1}$ Furthermore, about $90 \%$ of the Indigenous users reported symptoms of cannabis dependence. ${ }^{1}$ This compares with about $20 \%$ of users aged 18 or over in the general Australian population. ${ }^{3}$ Of even greater concern was a suggestion that, for most Indigenous users, cannabis was not a passing adolescent phase. After 5 years of follow-up, the great majority reported continuing heavy use. ${ }^{4}$

Cannabis use was linked to substantial health problems and social burdens in these communities, which are already disadvantaged by isolation and poverty. ${ }^{2,5,8} \mathrm{Up}$ to $10 \%$ of the communities' total income and between $31 \%$ and $62 \%$ of a user's median weekly income was spent on cannabis. ${ }^{5}$ Cannabis users were less likely than non-users to participate in education or training ${ }^{5}$ and more likely to report auditory hallucinations, suicidal ideation, ${ }^{6}$ symptoms of depression, ${ }^{7}$ and having been imprisoned. ${ }^{6}$ Community violence increased when cannabis supplies were scarce. ${ }^{1,2}$ The effects on traditional life were described by one NT Indigenous mental health clinician in the following way:

Too many of my people are chained to [cannabis]. They don't go out hunting or spend time by the river with their family.

They just sit and smoke [cannabis], then look for money to buy

more [cannabis] and get into fights when they can't get any

(Muriel Jaragba, personal communication).

What accounts for the unusual patterns of cannabis misuse in these remote Indigenous communities? There is little evidence that cannabis is grown locally, ${ }^{9}$ but much anecdotal evidence that market networks supplied by dealers based in urban or regional centres are extensive and resilient, making cannabis readily available (A R C, unpublished observation). Alcohol restrictions have been effective in reducing problem drinking within communities, but may have had the undesirable consequence of encouraging an increase in cannabis use where it could be easily obtained. ${ }^{5}$ As with risks for other forms of substance misuse in these communities, the social context is important. Limited employment and education opportunities; crowded, poor-quality housing; community-wide feelings of disempowerment; and grief and loss related to high mortality, morbidity and incarceration rates are all likely 


\section{EDITORIALS}

risk factors for substance misuse. Cannabis misuse is likely to be both a consequence of this type of social disadvantage and a perpetuating influence.

Cannabis misuse in remote Indigenous communities has been overlooked for too long. It is now clear that it is yet another major problem for these already disadvantaged communities, with evidence of cannabis misuse across a broad area of northern Australia. ${ }^{1,2,9}$ As well as in the NT, concerns about the level of cannabis use have recently been noted in Cape York ${ }^{8}$ and anecdotally in other parts of remote and regional Australia. Further research is needed to investigate the impact of cannabis use on urban Aboriginal and Torres Strait Islander Australians.

Effective responses will not be easy. Controls on supply by state- or territory-based police are one of the few available measures. ${ }^{6}$ In order to be effective, policymakers and service providers would need to work collaboratively with local communities to tie in local prevention and treatment initiatives with existing supply control initiatives. Such programs would need to use Indigenous language and cultural frameworks, build capacity of local Indigenous professionals, and improve understanding of the harms associated with cannabis misuse. ${ }^{10}$ Ultimately, tackling the misuse of cannabis and other substances in remote settings will depend on creating opportunities for social development and for continuing education, training and employment of adolescents and young adults.

\section{Acknowledgements}

We gratefully acknowledge the support of the National Health and Medical Research Council (NHMRC) and the Alcohol Education and Rehabilitation Foundation. Kylie Lee was supported by an NHMRC training scholarship for Indigenous Australian health research.

\section{Author details}

KS Kylie Lee, BMus(Hons), PhD Student, ${ }^{1}$ and Research Assistant ${ }^{2}$ Katherine M Conigrave, FAFPHM, FAChAM, PhD, Associate Professor, ${ }^{2}$ and Addiction Medicine Specialist ${ }^{3}$

George C Patton, MD, FRANZCP, Professor ${ }^{4}$

Alan R Clough, PhD, Associate Professor ${ }^{1}$

1 School of Public Health, Tropical Medicine and Rehabilitation Sciences, James Cook University, Cairns, QLD.
2 Faculty of Medicine, University of Sydney, Sydney, NSW. 3 Drug Health Service, Royal Prince Alfred Hospital, Sydney, NSW. 4 Centre for Adolescent Health, Murdoch Childrens Research Institute, University of Melbourne, Melbourne, VIC.

Correspondence: kylie.lee@usyd.edu.au

\section{References}

1 Lee KSK, Clough AR, Conigrave KM. High levels of cannabis use persist in Aboriginal communities in Arnhem Land, Northern Territory [letter]. Med J Aust 2007; 187: 594-595.

2 Select Committee on Substance Abuse in the Community, Legislative Assembly of the Northern Territory. Substance abuse in remote communities: confronting the confusion and disconnection. Darwin: Northern Territory Government, 2007. http://www.nt.gov.au/lant/parliament/committees/substance/Substance\%20Abuse\%20Report\%20CONTENT.pdf (accessed May 2008).

3 McLaren J, Mattick RP. Cannabis in Australia: use, supply, harms and responses. Canberra: Drug Strategy Branch, Australian Government Department of Health and Ageing, 2007. (Monograph Series No. 57.) http://www.nationaldrugstrategy.gov.au/internet/drugstrategy/publishing.nsf/Content/mono57 (accessed Apr 2008).

4 Lee KSK, Clough AR, Conigrave KM, et al. Five year longitudinal study of cannabis users in three remote Aboriginal communities in Arnhem Land, Northern Territory, Australia. Drug Alcohol Rev 2008. In press.

5 Clough AR, d'Abbs P, Cairney S, et al. Emerging patterns of cannabis and other substance use in Aboriginal communities in Arnhem Land, Northern Territory: a study of two communities. Drug Alcohol Rev 2004; 23: 381-390.

6 Clough AR, Lee KSK, Cairney S, et al. Changes in cannabis use and its consequences over 3 years in a remote Indigenous population in Northern Australia. Addiction 2006; 101: 696-705.

7 Lee KSK, Clough AR, Jaragba MJ, et al. Heavy cannabis use and depressive symptoms in three Aboriginal communities in Arnhem Land, Northern Territory. Med J Aust 2008; 188: 605-608.

8 Robertson J, Dowie R. Cannabis: a cloud over our community. Of Substance 2008; 6: 28-29. http://ofsubstance.org.au/archive/pdf/ ofsubstance_2008_7.pdf\#article13 (accessed Aug 2008).

9 Delahunty B, Putt J. The policing implications of cannabis, amphetamine and other illicit drug use in Aboriginal and Torres Strait Islander communities. Canberra: Australian Institute of Aboriginal and Torres Strait Islander Studies and Australian Institute of Criminology, 2006. (Monograph Series No. 15.) http://www.aic.gov.au/publications/other/2006ndlerfmono15.html (accessed Jun 2006).

10 Lee KSK, Jaragba MJ, Clough AR, Conigrave KM. Wa! Ningeningma arakba akina da! (Oh! Now I know, that's it!). Providing feedback to communities about studies of cannabis use in Arnhem Land, Northern Territory. Med J Aust 2008; 188: 113-116. 